\author{
Military Technical College \\ Kobry Elkobbah, Cairo, \\ Egypt.
}

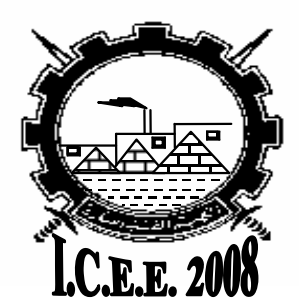

\author{
$4^{\text {th }}$ International Conference On \\ Chemical \& Environmental \\ Engineering
}

27-29 May 2008

\title{
POLYANILINE: A CONDUCTING POLYMER
}

\author{
M. M. AYAD*
}

\begin{abstract}
Conducting polyaniline (PANI) is one of the most intensively investigated polymers during the last years. This is due to its environmental stability, high degree of processability and interesting redox properties associated with its chain. PANI can be obtained by chemical or electrochemical polymerization of aniline in the forms of a powder in bulk of solution or a deposited film. However a great deal of attention was paid in recent years to the deposition of a thin film of PANI on various surfaces. These films have great importance in many technological applications. Therefore the presentation is devoted to discuss: firstly, the chemical polymerization of aniline with suitable oxidant to obtain the polymer with desired properties. Secondly, the polymerization kinetics, conductivity, dopant, medium and the presence of some additives on the polymer film formation are discussed. In addition the application of these films as $\mathrm{pH}$, acid and pollutant sensors are mentioned. Finally, the inclusions of these polymers into clay to obtain useful nanocomposite materials are outlined.
\end{abstract}

\footnotetext{
* Department of Chemistry, Faculty of Science, University of Tanta, Tanta, Egypt
} 